\title{
Spacecraft potential control aboard Equator-S as a test for Cluster-II
}

\author{
K. Torkar ${ }^{1}$, W. Riedler ${ }^{1}$, M. Fehringer ${ }^{2}$, F. Rüdenauer ${ }^{2}$, C. P. Escoubet ${ }^{3}$, H. Arends ${ }^{3}$, B. T. Narheim ${ }^{4}$, \\ K. Svenes ${ }^{4}$, M. P. McCarthy ${ }^{5}$, G. K. Parks ${ }^{5}$, R. P. Lin ${ }^{6}$, and H. Rème ${ }^{7}$ \\ ${ }^{1}$ Space Research Institute, Austrian Academy of Sciences, Inffeldgasse 12, A-8010 Graz, Austria \\ E-mail: klaus.torkar@oeaw.ac.at \\ ${ }^{2}$ Austrian Research Centre Seibersdorf, A-2444 Seibersdorf, Austria \\ ${ }^{3}$ Space Science Division, ESA/ESTEC, NL-2200 AG Noordwijk, The Netherlands \\ ${ }^{4}$ Norwegian Defence Research Establishment, N-2007 Kjeller, Norway \\ ${ }^{5}$ University of Washington, Seattle, WA 98195 , USA \\ ${ }^{6}$ Space Sciences Laboratory, University of California, Berkeley, CA 94720, USA \\ ${ }^{7}$ CESR, Université Paul Sabatier, BP 4346, F-31028 Toulouse, France
}

Received: 8 March 1999 / Revised: 15 July 1999 / Accepted: 20 July 1999

\begin{abstract}
The payload of Equator-S was complemented by the potential control device (PCD) to stabilise the electric potential of the spacecraft with respect to the ambient plasma. Low potentials are essential for accurate measurements of the thermal plasma. The design of PCD is inherited from instruments for Geotail and Cluster and utilises liquid metal ion sources generating a beam of indium ions at several $\mathrm{keV}$. The set-up of the instrument and its interaction with the plasma instruments on board is presented. When the instrument was switched on during commissioning, unexpectedly high ignition and operating voltages of some ion emitters were observed. An extensive investigation was initiated and the results, which lead to an improved design for Cluster-II, are summarised. The cause of the abnormal behaviour could be linked to surface contamination of some emitters, which will be monitored and cured by on-board procedures in future. The mission operations on Equator-S were not at all affected, because of the high redundancy built into the instrument so that a sufficient number of perfectly operating emitters were available and were turned on routinely throughout the mission. Observations of the effect of spacecraft potential control on the plasma remained limited to just one event on January 8, 1998, which is analysed in detail. It is concluded that the ion beam lead to the predicted improvement of the particle measurements even outside the low density regions of the magnetosphere where the effect of spacecraft potential control would have been much more pronounced, and that the similar instruments for the four Cluster-II spacecraft to be launched in 2000 will be very important to ensure accurate plasma data from this mission.
\end{abstract}

Key words. Space plasma physics (active perturbation experiments; spacecraft sheaths, wakes, charging; instruments and techniques)

Correspondence to: $\mathrm{K}$. Torkar

\section{Introduction}

Despite being a small spacecraft of less than $235 \mathrm{~kg}$ dry mass, Equator-S carried a complete payload for plasma and field investigations in the Earth's magnetosphere. Even though long wire booms could not be accommodated and, hence electric field and spacecraft potential measurements in the traditional double probe technique were impossible, electric field data were obtained by the electron drift instrument EDI (Paschmann et al., this issue). The plasma measurements were covered by the 3D-plasma analyser (3DA) instrument, based on electrostatic analysers for electrons and ions in the energy range from a few $\mathrm{eV}$ to $25 \mathrm{keV}$, and the ion composition instrument (ESIC) which could resolve the major ion species in the E/q range from essentially zero up to $40 \mathrm{keV}$. Particles at higher energies were measured by the energetic particle instrument (EPI). With this comprehensive set of particle instruments, Equator-S was designed to provide plasma data in a quality similar to that of the large spacecraft that complement the Inter-Agency Solar Terrestrial Science Programme (IASTP), in particular Geotail, Wind, Polar, Interball, and the future four Cluster-II spacecraft. The high spin rate of Equator-S (nominal spin period $1.5 \mathrm{~s}$ ) laid special emphasis on resolving temporal features, while the small resources for the payload dictated some compromises with respect to other parameters.

Nevertheless, Equator-S was in need of a device to control the electric potential of the spacecraft with respect to the ambient plasma. The rationale for active spacecraft potential control has been established in the early 1980s (see, e.g. Pedersen et al., 1983) and has ultimately lead to the installation of active control devices on many IASTP spacecraft: Geotail (Schmidt et al., 1995), Interball-2 (Torkar et al., 1998), Polar (Moore et al., 1995), and the forthcoming Cluster-II (Riedler et al., 1997).

Potentials of up to several tens of volts impede the measurement of the core of the distribution functions of 
the plasma and make it difficult or impossible to derive fundamental parameters such as density, temperature and flow velocity (see, e.g. Olsen, 1982). Positive potentials also trap a large fraction of the photoelectrons that are inevitably created at the sunlit spacecraft surfaces in the sheath around the spacecraft. The photo-electrons then dominate the low-energy portion of the measured spectra, causing substantial difficulties in interpreting the data and restricting any automatic calculation of moments.

Potential drops between the ambient plasma and a spacecraft result from an equilibrium of spacecraftplasma interactions under the influence of solar radiation and not only involve the thermal plasma, but also energetic particles. The potential differences are kept to a minimum when good conductivity between the spacecraft and its environment is maintained even in a rarefied plasma. Respective methods which have been implemented on spacecraft comprise the emission of a quasi-neutral artificial plasma cloud around the spacecraft, as on the Polar spacecraft, and the emission of positive ions at energies of several kilo-electron-volts, as on Geotail and Interball-2. Whereas the plasma source technique can cope with both positive and negative charging of a spacecraft, beams of positive ions obviously can only remove positive potentials. Plasma sources, however, under some circumstances induce a major disturbance of the spacecraft environment that e.g. promotes the growth and propagation of plasma waves. Plasma sources as on Polar and ion sources based on gas discharge as on Interball-2 furthermore require heavy tanks for the source material. This is not the case for a new generation of ion emitters, the working principle of which is based on the ionisation of a film of liquid metal in a strong electric field. These liquid metal ion sources (LMIS) are characterised by a small mass which is almost negligible in comparison to the required housing for protection and drive electronics. They have been successfully implemented in an instrument aboard the Geotail spacecraft (EFD-iE) and have also been selected for Equator-S as well as for the four forthcoming Cluster-II spacecraft.

The rationale for choosing LMIS devices for Geotail, Cluster-II, and Equator-S does not only rely on their small mass and power requirements, but is also linked to the scientific objectives of the missions. Geotail and Cluster-II have orbits that almost always avoid regions in the Earth's magnetosphere where a spacecraft would get charged negatively, such as in the plasmasphere when the density exceeds some hundred particles per $\mathrm{cm}^{3}$, the radiation belts, and the equatorial region at geosynchronous distance, where substorm-related particle events may cause transient negative charging to several kilovolts, particularly in eclipse. The uncontrolled potential of Geotail and Cluster-II is or will be in general positive, and even rise to several tens of volts in the lobes and in polar regions. Equator-S with its eccentric equatorial orbit (apogee at 11.5 $\mathrm{R}_{\mathrm{E}}$ geocentric distance, perigee at $500 \mathrm{~km}$ altitude) flew through a great variety of plasma conditions, but the main scientific objectives were focused on regions outside the radiation belts and the plasmasphere. Thus, also Equator-S was served well by a device capable of reducing positive charging of the spacecraft. Equator-S also supported technological research by carrying several dedicated instruments. The technological aspects of a potential control device based on ion emitters were therefore part of the mission objectives. The Equator-S team always maintained an experimental attitude which permitted investigations of spacecraft-plasma interactions by energetic ion beams to a greater extent than aboard the major IASTP missions, where continuous measurements of the magnetospheric plasma have virtually absolute priority over experiments on ion beam effects.

We have a dual objective to demonstrate the working principle of the applied technique under conditions encountered at the orbit of Equator-S, which is quite different from those of the spacecraft Geotail and Polar, and to show which lessons were learned for future missions by operating the potential control device.

\section{Instrumental set-up}

The application of liquid metal ion sources for spacecraft potential control has been described in the literature before in the context of the missions Geotail (Schmidt et al., 1993) and Cluster-II (Riedler et al., 1997). The basic principle (see, e.g. Mahoney et al. 1969) finds widespread application in laboratories. After a major redesign the ion sources were qualified for space use (for the current state of development see Fehringer et al., 1997). In that design a sharpened tungsten needle is mounted in the centre of a cylindrical indium reservoir. During operation the indium in the reservoir is molten and a thin indium film covers the needle. A high voltage of about $6 \mathrm{kV}$ is applied between the emitter tip and an accelerator electrode which is mounted opposite to the tip. Due to the small radius of curvature at the tip apex the local electric field reaches values in the order of volts per nanometre, high enough to enable field ion emission. Indium (stable isotopes with $115 \mathrm{amu}$ and $113 \mathrm{amu}$ to $95.7 \%$ and $4.3 \%$, respectively) has been chosen as the ion-source charge material because of its low vapour pressure, which prevents contamination of the source insulators and ambient spacecraft surfaces. On the other hand, the melting point at $156.6{ }^{\circ} \mathrm{C}$ is high enough to prevent melting of an unheated source charge. Rüdenauer et al. (1987) showed that the beam consists of $>90 \%$ single-charged $\mathrm{In}^{+}$with minor fractions of double- and triple-charged ions and single-charged indium clusters. Depending on the size of the reservoir, up to $4000 \mathrm{~h}$ nominal operating time at $10 \mu \mathrm{A}$ emission current can be achieved with a single emitter. In order to extend the operating time of the instrument and to provide additional redundancy, eight emitters were combined into one instrument, grouped into two emitter modules powered by separate supplies. Focusing electrodes shape the beam into a cone of $15^{\circ}$ half width, half maximum. Due to the low power consumption (only $\approx 0.5 \mathrm{~W}$ of heater power to keep the 
indium in molten state in addition to the energy of the beam) and the small mass of the sources (a single emitter weighs one gram; four emitters combined into a module with electrodes and housing weigh $180 \mathrm{~g}$ ) the total mass of the instrument including housing, electrical supplies, the control unit, and $0.8 \mathrm{~kg}$ additional radiation shielding (compared to Cluster-II or Geotail) to allow frequent passages through the radiation belts is $2.7 \mathrm{~kg}$. The maximum power consumption including electronics is $2.7 \mathrm{~W}$.

The potential control device (PCD) for Equator-S has been inherited from instruments for the Geotail and original Cluster missions (Schmidt et al., 1993; Riedler et al., 1997). Since Geotail (launched in 1992) the capacity of the indium reservoir was increased from about 80 to $200 \mathrm{mg}$, increasing the theoretical lifetime by a factor of 2.5. At the same time the number of emitters per module was reduced from 5 to 4 giving space to introduce shielding between the individual ion sources. This shielding was meant to prevent sputter particles from deposition on adjacent needles. In total the theoretical instrument lifetime was increased to $32000 \mathrm{~h}$ by these changes. Finally the available maximum high voltage was raised from 7.5 to $8.3 \mathrm{kV}$. The outer appearance of the instrument (Fig. 1) was identical to the instrument ASPOC (active spacecraft potential control) for Cluster apart from the mechanical interface to the thermal blanket of the spacecraft. The characteristic parameters of the instrument are summarised in Table 1. The instrument was mounted at the subsystem platform of Equator-S, and the ion beam pointed into the direction of the spin axis.

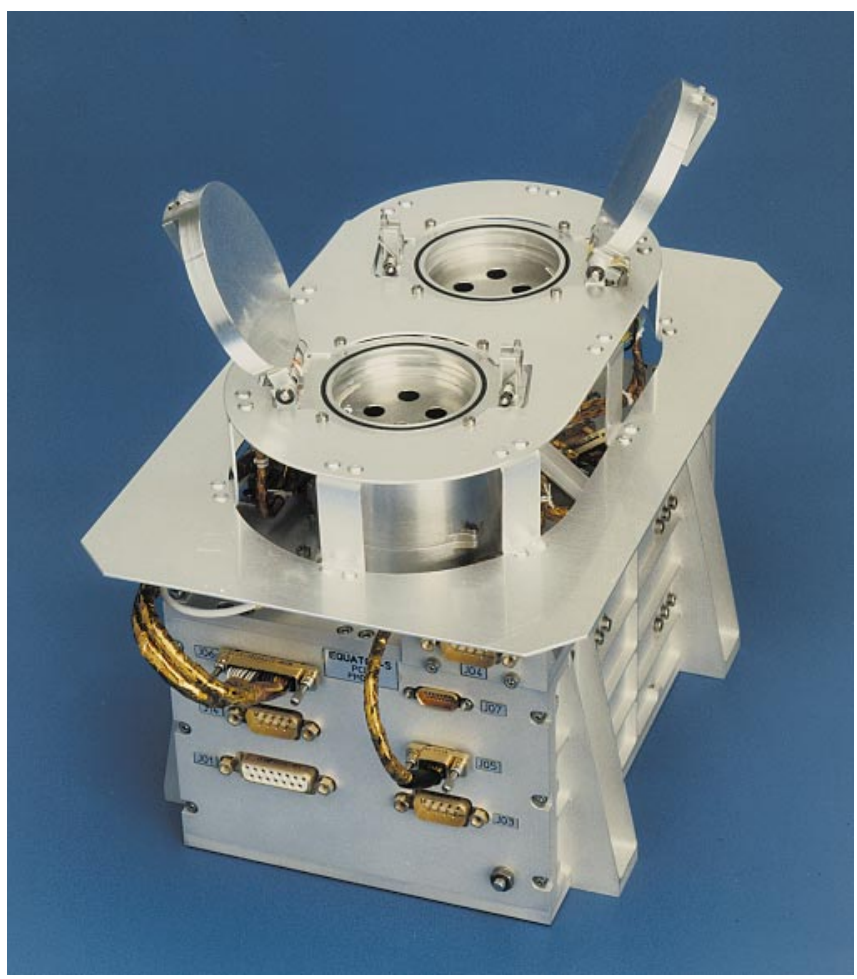

Fig. 1. Potential control device for Equator-S
Table 1. Parameters of the instrument PCD

\begin{tabular}{ll}
\hline Mass & $2700 \mathrm{~g}$ \\
\hline Size & \\
Electronics box & $187 \times 157 \times 95 \mathrm{~mm}$ \\
Emitter modules & $60 \mathrm{~mm}$ diameter $\times 75 \mathrm{~mm}$ \\
Overall & $187 \times 157 \times 170 \mathrm{~mm}$ \\
Power & $2.4 \mathrm{~W}$ average. $2.7 \mathrm{~W}$ peak \\
Telemetry rate & $128 \mathrm{bit} / \mathrm{s}$ \\
Design lifetime & $32,000 \mathrm{~h}$ at $10 \mu \mathrm{A}$ \\
Beam & \\
Species & $\mathrm{In}^{+}$ \\
Atomic mass & 113,115 amu \\
Energy & 5 to $8 \mathrm{keV}$ \\
Current & Maximum: $50 \mu \mathrm{A}$ \\
& Design: $10 \mu \mathrm{A}$ \\
Opening angle & $\pm 15^{\circ}$ (half maximum) \\
Direction & Along spin axis \\
\hline
\end{tabular}

The 3D plasma analyser instrument 3DA (together with the instrument EPI) is a derivative of the $3 \mathrm{D}$ plasma and energetic particle instrument on the Wind spacecraft. It consists of an electron electrostatic analyser (EESA) and an ion electrostatic analyser (PESA). The analyser is a symmetrical spherical-section electrostatic analyser in top-hat design with a disk-shaped field of view of $180^{\circ}$, directed perpendicular to the spacecraft body. The analysers measured distribution functions in 192 angle and 56 energy channels. The data presented in a later section have been taken from the lowermost 13 energy channels with centre energies ranging from 7.5 to $98.8 \mathrm{eV}$.

A special feature of the hardware set-up, which unfortunately could not be tested aboard Equator-S, was an on-board data link between the instruments 3DA and PCD. At intervals of about one minute 3DA transmitted a subset of measured count rates (only for energies below $100 \mathrm{eV}$ and for selected angular sections) to PCD. The processor of PCD has been programmed to search for structures within the transmitted spectra which are related to the potential of the spacecraft. Assuming that the flux of electrons out of the ambient plasma is small in comparison to the total flux of photoelectrons generated at the surface, one would expect a ledge in the energy spectrum near the spacecraft potential. Below a critical energy photo-electrons would dominate the flux, whereas all plasma electrons would have energies above the threshold since they experience a net increase of energy during their passage through the sheath. Although this description may be oversimplified, more thorough analyses of the potential structures around a spacecraft with potential control (e.g. Whipple, 1976; Zhao et al., 1996) confirm this basic scenario.

Having identified the spacecraft potential in the electron spectrum, the ion current emitted by PCD would be adjusted to maintain a constant potential in a closed control loop. The difference between the real spacecraft potential and the energy where spectral features due to the spacecraft potential appear could be neglected, as the main purpose of the control was to achieve a low and stable, but not necessarily zero, potential. The instrument 3DA, having a lower energy limit of $\approx 7.5 \mathrm{eV}$, did not require exactly zero potential. 
It must be emphasised that the stability of the controlled potential is entirely sufficient even without the closed loop approach just described. Schmidt et al. (1995) have demonstrated by analysing data from Geotail that an ion beam with a constant current of $15 \mu \mathrm{A}$ clamped the potential of this spacecraft to less than $4 \mathrm{~V}$ positive even in the outer lobes of the magnetosphere where the uncontrolled potential reached about $50 \mathrm{~V}$.

\section{Instrument operation aboard Equator-S}

On December 13, 1997, PCD was operated in space for the first time. All mechanisms, in particular the protective covers of the emitter modules which had been opened by pyrotechnic actuators after launch, electronics, and software worked without flaws. Four of the eight emitters with nominally $4000 \mathrm{~h}$ operation time each fired, the remaining four did not. The failure of two emitters was no surprise, as they had already shown deviations from nominal behaviour on the ground. This had been accepted since only two emitters would have been sufficient to cover two years of flight operations. The failure of the other two emitters was unexpected as they had been working very well on the ground. A possible but not verifiable explanation would be a failure in the hermetic sealing system that might have led to the introduction of humid air. The investigations carried out on that matter are described later.

During further tests in the commissioning phase, one of the four operational emitters (A3) was permanently improving in operating stability and it was decided to use that emitter as a working horse until results from laboratory measurements would be available that might help to start some of the emitters which could not be activated in the first round of tests. After the end of the commissioning phase the instrument PCD was operated on a routine basis for almost four months from January until the end of April, 1998, when the spacecraft processor failed. A typical operation cycle of PCD during this exploration phase of the mission included one-hour on/off periods to identify the effects on the plasma measurements, as shown in Fig. 2. After an initial heating phase of $\approx 15 \mathrm{~min}$, when the emitter temperature is slowly raised to operating level $\left(\approx 230{ }^{\circ} \mathrm{C}\right)$, high voltage is turned on and is increased smoothly until the emitter ignites. After ignition the control of the ion current takes over. Due to some limitations of the total number of commands that could be stored in the time-tagged queue not more than two turn-on periods per orbit, separated by one hour of hot standby, were possible. In hot standby the indium in the reservoir is kept in molten state, so that re-starting the emitter is achieved within seconds.

The nominal ion current of $12 \mu \mathrm{A}$ was very stable throughout the mission. Short-time variations remained below the detection limit of $0.2 \mu \mathrm{A}$. At the end of each operating cycle the current was increased to $\approx 50 \mu \mathrm{A}$ over $1 \mathrm{~min}$ in order to exploit the capabilities of the device and as a precaution against contamination of the source. It is well known from laboratory use of liquid metal ion sources that short impulses at high emission current can remove obstacles in the liquid flow that might have accumulated. The attempt to assess the longterm behaviour of an emitter over a much longer period was, however, terminated by the early failure of the spacecraft. The operating voltage of the emitter at $12 \mu \mathrm{A}$ current was $6.5 \mathrm{kV}$ and increased only marginally to $6.75 \mathrm{kV}$ at $50 \mu \mathrm{A}$.

Emitter A3 had been operated for about $250 \mathrm{~h}$ in total and showed perfect behaviour until the premature failure of the spacecraft. Ignition and operational voltages essentially remained unchanged throughout the mission.

\section{Operating parameters of the ion emitters}

In spite of the flawless operation of the selected emitter throughout the mission the failure of some other emitters triggered an extensive investigation, aiming at

\section{Equator-S Potential Control Device}

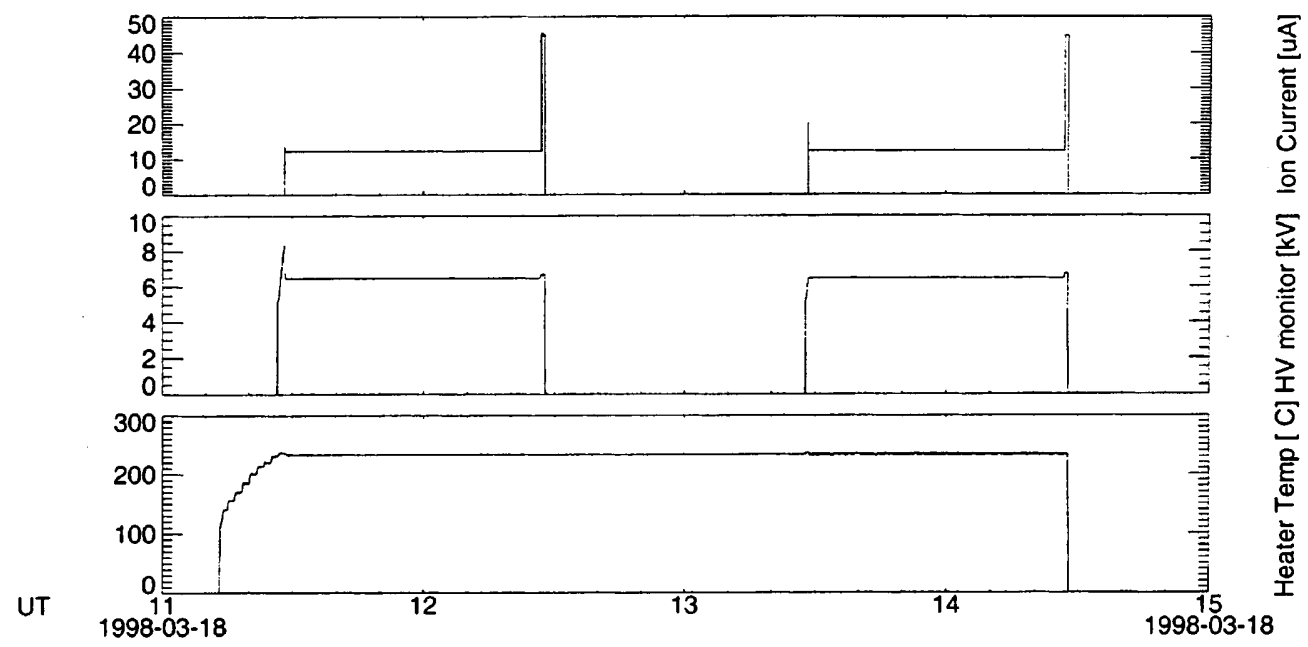

Fig. 2. Routine operation of the instrument PCD. From top to bottom: ion current, operating voltage, and heater temperature 
identifying the causes for the apparent increase of the ignition voltage beyond the capabilities of the electrical supply. The assumption that contamination of indium tips by particles sputtered from electrodes leads to elevated ignition voltages was to be tested. Ion emitters that have been exposed to intense sputtering were analysed in a high position-sensitive electron microprobe. Surface micrographs for typical contamination elements like iron, chromium, and nickel, i.e. the main constituents of the stainless steel electrodes, were recorded along the surface of an indium needle. In addition, relative quantities of the respective contaminants were determined on selected locations. Figure 3 shows a micrograph from the very end of an indium tip with a lateral resolution in the $1 \mu \mathrm{m}$ range. Two distinct locations, where elementary analysis has been performed, are indicated together with the relative contributions in particle numbers of the investigated elements. It can easily be seen that in addition to areas covered with pure indium plate-like structures with high concentrations of iron and chromium exist. Indium is rich at the tip apex indicating a functional ion emitter.

In parallel to the investigations of material deposited on the indium film also the ion beam was analysed for components other than indium. A double-focusing, four-sector mass spectrometer with a dynamic range of nine orders of magnitude was employed to perform these measurements. All typical constituents of stainless steel that have already been detected on the needle surface in the electron microprobe measurements are also found in the beam. Typical concentrations of the two most prominent contamination elements, iron and chromium, are $24 \mathrm{ppm}$ and $7 \mathrm{ppm}$ respectively. In addition, the beam contained very small amounts of impurities which were present already in the pure indium as delivered from the manufacturer.

The results of these described measurements support the following scenario: sputter products from either the beam-forming electrodes or the walls of the vacuum chamber are deposited on the indium film that covers the tungsten needle of the emitter. Parts of these deposits are re-emitted via field emission as are the indium atoms and can be found in the beam. The other part of the sputter products accumulate on the needle surface, freeze out locally and form small plates. These plates may be taken with the liquid film flow towards the very tip apex and cause blockage. During emitter operation it is often observed that the operating voltage slowly rises and very suddenly returns to its initial value. We interpret this increase in voltage as a need for more stress to maintain the liquid cone which again can be caused by frozen plates blocking the indium flow. With

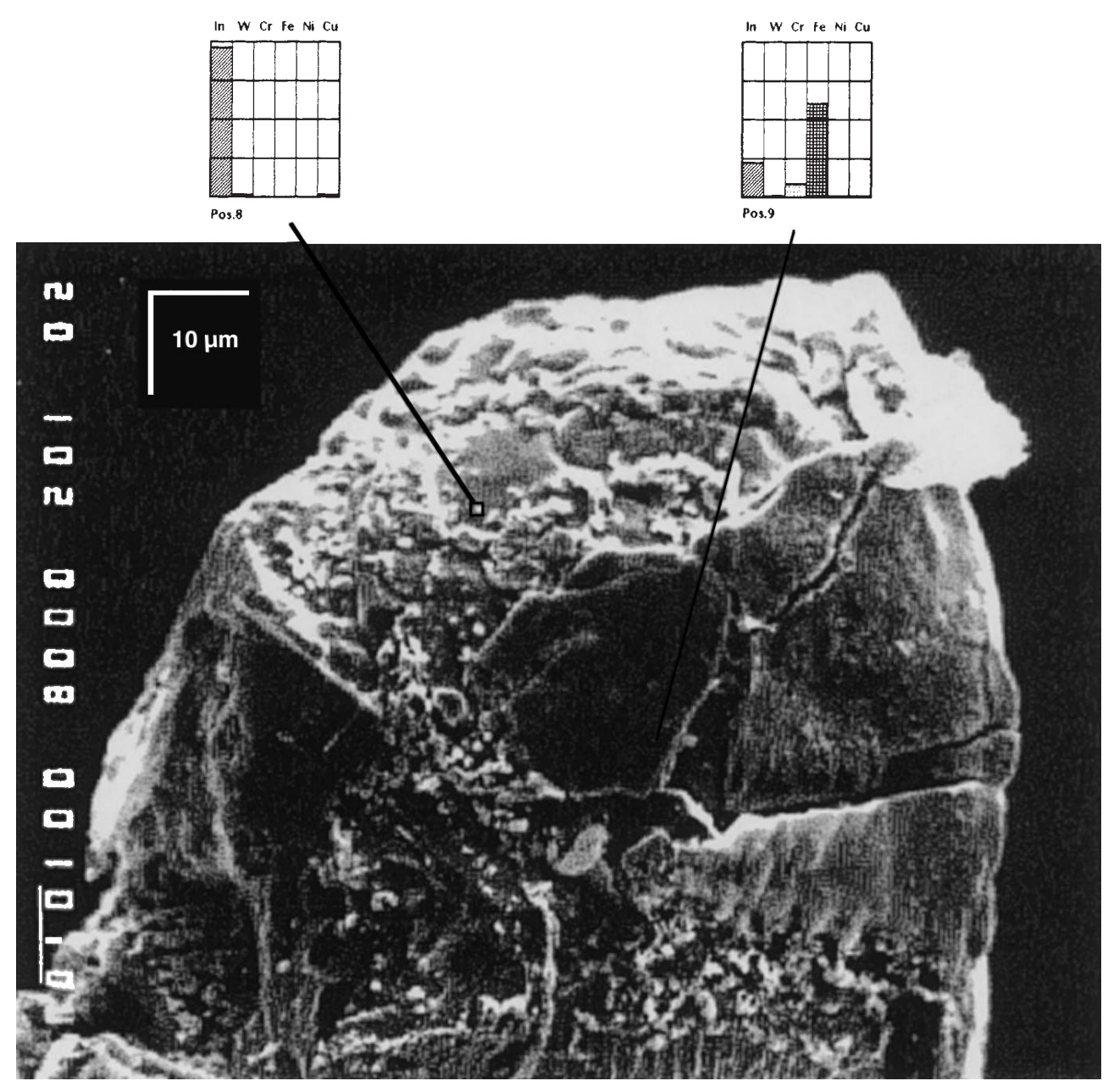

Fig. 3. Image of an indium emitter tip from an electron microscope. At two selected areas elementary analysis has been performed. Relative particle numbers of elements are given in the bar graphs at the top 
increasing voltage and increasing force on the liquid tip these plates may be cleared away and the voltage drops instantly. This explanation is supported by the fact that such plates disappear sometimes from one operational period to the next.

This explanatory model holds for an operating emitter. As long as an emitter is running it hardly ever fails. Its ignition voltage at re-start does not differ significantly from the last operational voltage as long as the vacuum is not broken and no adjacent emitter is activated. Obviously, enough sputter particles are reemitted in the beam in order to keep the tip apex clean. This is different in the case of non-operating ion sources. During operation of the adjacent emitter sputter particles are deposited and there is no mechanism to remove them. They accumulate and form a film that has a considerably higher melting point than the pure indium film. During ignition, sometimes a considerably higher force, meaning higher ignition voltage, has to be applied in order to break up that film and expose the pure indium to the electric field. Once the emitter has then ignited it usually instantly returns to its original operational voltage.

\section{Lessons learned for Cluster-II}

The four Cluster-II spacecraft will be equipped with instruments which are very similar to PCD since this instrument was based on the design and some spare parts from the first four Cluster spacecraft, which had been destroyed in the launch failure of Ariane-501. Therefore the experience from Equator-S was extremely valuable and helped to improve hardware, software, and flight operations. The technical experience with the ion emitters aboard Equator-S can be summarised as follows. Among the design changes that had evolved since Geotail in 1992, i.e. a bigger reservoir and a thicker indium film on the needles, the latter appeared to have both positive and undesirable effects on the performance. While the thicker film helps to raise the maximum current of the emitter and to reduce the associated voltage increase, the larger tip radius associated with a thick layer of indium may also lead to a higher ignition voltage, particularly in the presence of contamination sources. The improvements for the similar instrument to be flown aboard Cluster-II (ASPOC) are related to the emitters, the electrical supply, and the control software.

1. The thickness of the indium film is monitored and tailored during the wetting process. Thick layers of indium are avoided.

2. Further shielding of emitters against backsputtering from walls of the vacuum chamber is applied in laboratory testing.

3. The capability of the high voltage supply is increased from 8.3 to $9.5 \mathrm{kV}$ to allow higher start-up voltages if needed. During nominal operations however, the voltage is limited by the software to $7.5 \mathrm{kV}$.

4. The changes of the instrument software essentially comprise the introduction of a threshold value for the high voltage, which, if exceeded, triggers either the shutdown of the high voltage or an automatic "cleaning" of the ion emitter by applying high current $(50$ to $80 \mu \mathrm{A})$ for 20 or $60 \mathrm{~s}$. This procedure is known to remove obstacles to the indium flow in the emitter (e.g. by an oxide layer). Afterwards the high voltage should return to a normal level.

5. For Cluster-II also regular tip cleaning cycles, which are not triggered by voltage increases, will be included in the flight operations plan as a precautionary measure. These events will be placed at the beginning or end of data acquisition periods.

The effect of the new cleaning procedure has been tested in the laboratory at an emitter which had been used extensively before and exhibited some variability of the operating voltage. Figure 4 shows the ion beam and voltage recordings. The current is kept at a constant level of $3.5 \mu \mathrm{A}$. When the contamination at the tip increases the voltage rises due to the higher electric impedance. As soon as $5.5 \mathrm{kV}$ are reached the on-board software initiates a current pulse of 70 or $50 \mu \mathrm{A}$ (this parameter was changed during the test). One can see that the voltage returns to normal values immediately after the current pulse for typically several hours. The voltage trigger level had been lowered to $5.5 \mathrm{kV}$ for demonstration purposes during this test only. In flight this level will be higher. Voltage and current during a single pulse are shown in detail in Fig. 5. The immediate effect on the operating voltage is apparent.

\section{Results and discussion of spacecraft potential control}

The plasma instruments aboard Equator-S, 3DA and ESIC, were intended to profit most from the spacecraft potential control. For ESIC, it was unfortunate that measurements in the retarding potential analyser mode did not have priority during the first months of the mission. The RPA would have lowered the energy window down to essentially zero volts, in which case the spacecraft potential control would have been mandatory for obtaining undisturbed data. Studies of charging effects on the thermal ion distribution functions would have been possible. A decision to start routine operations in RPA mode in May, 1998, came too late.

The instrument 3DA was measuring electron distribution functions simultaneously with the operation of the potential control device for the first time over several hours on January 8, 1998, as part of the commissioning of these instruments. Unfortunately this period remained the only source of data on the effect of spacecraft potential control on the plasma electrons, as the 3DA electron detector failed soon afterwards. Nevertheless, the advantageous effect of PCD on the electron measurements by controlling the spacecraft potential was verified successfully on that orbit.

In the late morning hours MLT of January 8, 1998, the ion beam of PCD had been turned on for several hours for check-out purposes, using a beam current of $12 \mu \mathrm{A}$. There was a short, 5-min interruption of the emission from 11:23 to 11:28 UT. The instrument 3DA 
new ASPOC software test current increase triggered at tip voltage $>5.5 \mathrm{kV}$

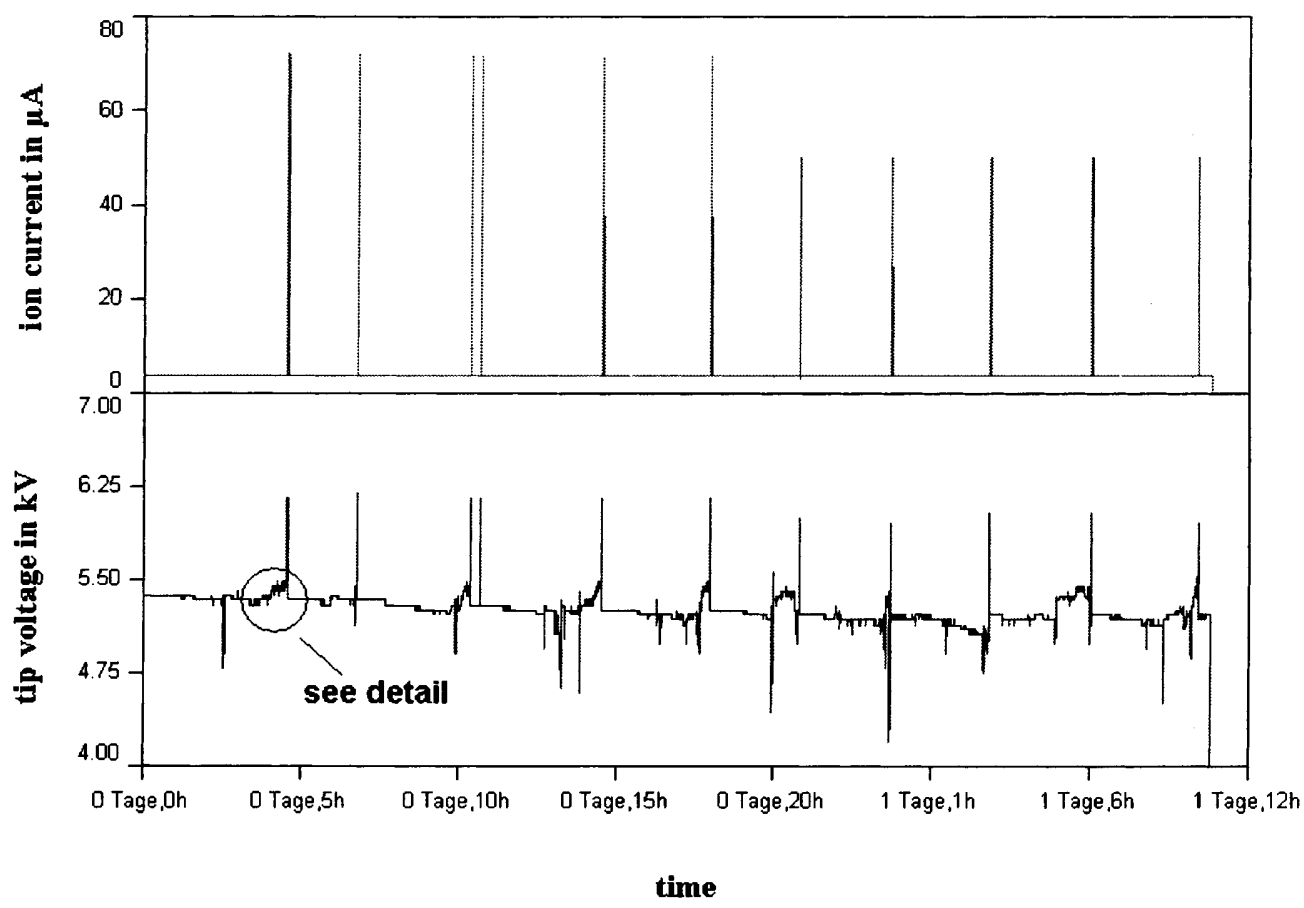

DETAIL 1

new ASPOC software test current increase triggered at tip voltage $>5.5 \mathrm{kV}$

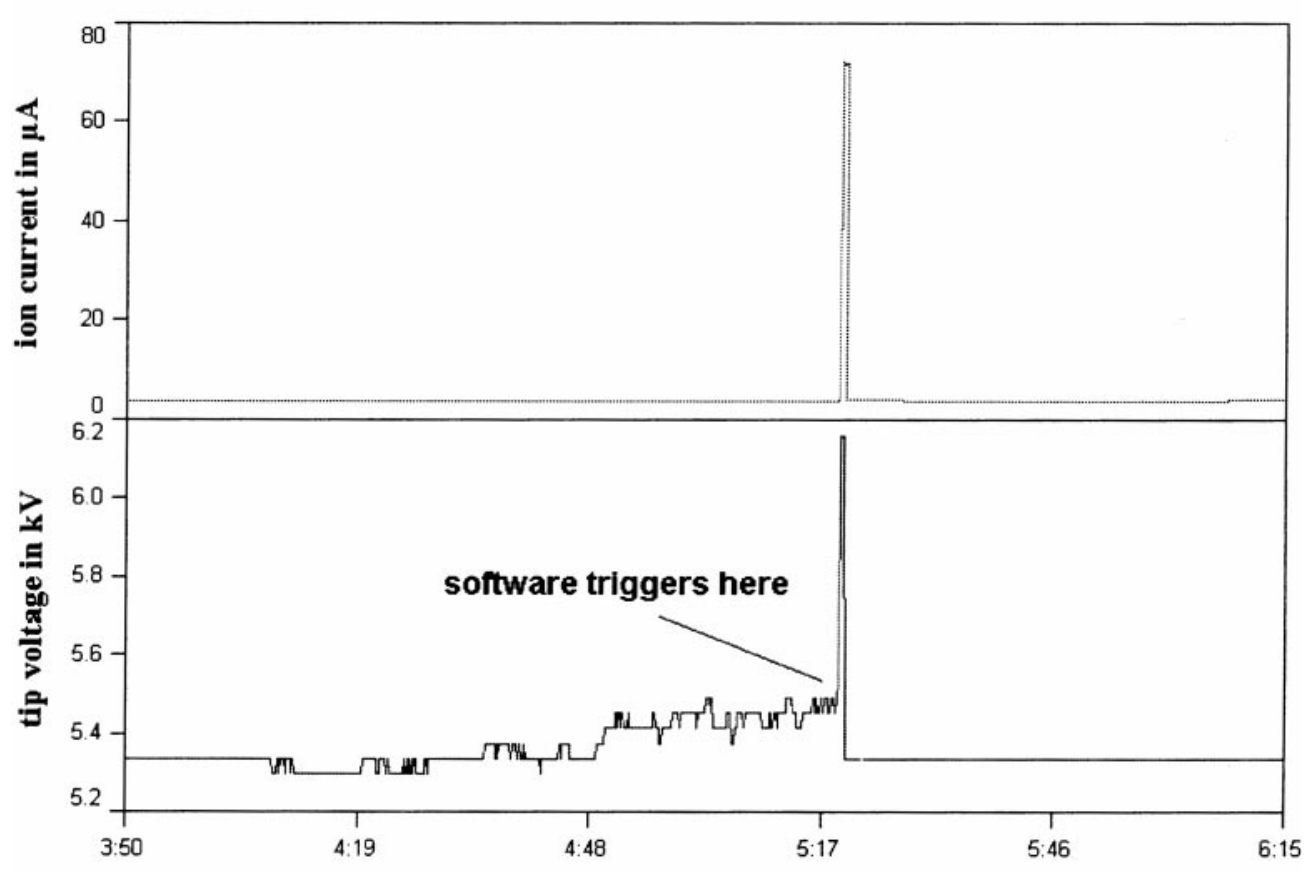

Fig. 4. Test of software for tip cleaning. Upper panel: ion current pulses are triggered by (lower panel) voltage increases above $5.5 \mathrm{kV}$
Fig. 5. Detail of Fig. 4 for a single cleaning cycle

time in hours : $\min$

had turned on the electron section and was measuring continuously. The differential number fluxes of the lowermost 13 energy bins $(7.5,9.2,11.3,14,17.1,21.1$, $26.2,32.7,40.5,50.3,62.5,77.8,98.8 \mathrm{eV})$ in the time interval 11:15 to 11:45 UT are shown in Fig. 6. During that time Equator-S was moving inbound from 8.1 to $7.5 \mathrm{R}_{\mathrm{E}}$ geocentric distance at $\approx 11 \mathrm{~h} \mathrm{LT}$, a location 3 to 3.5 $\mathrm{R}_{\mathrm{E}}$ inside the nominal magnetopause. The preceding 


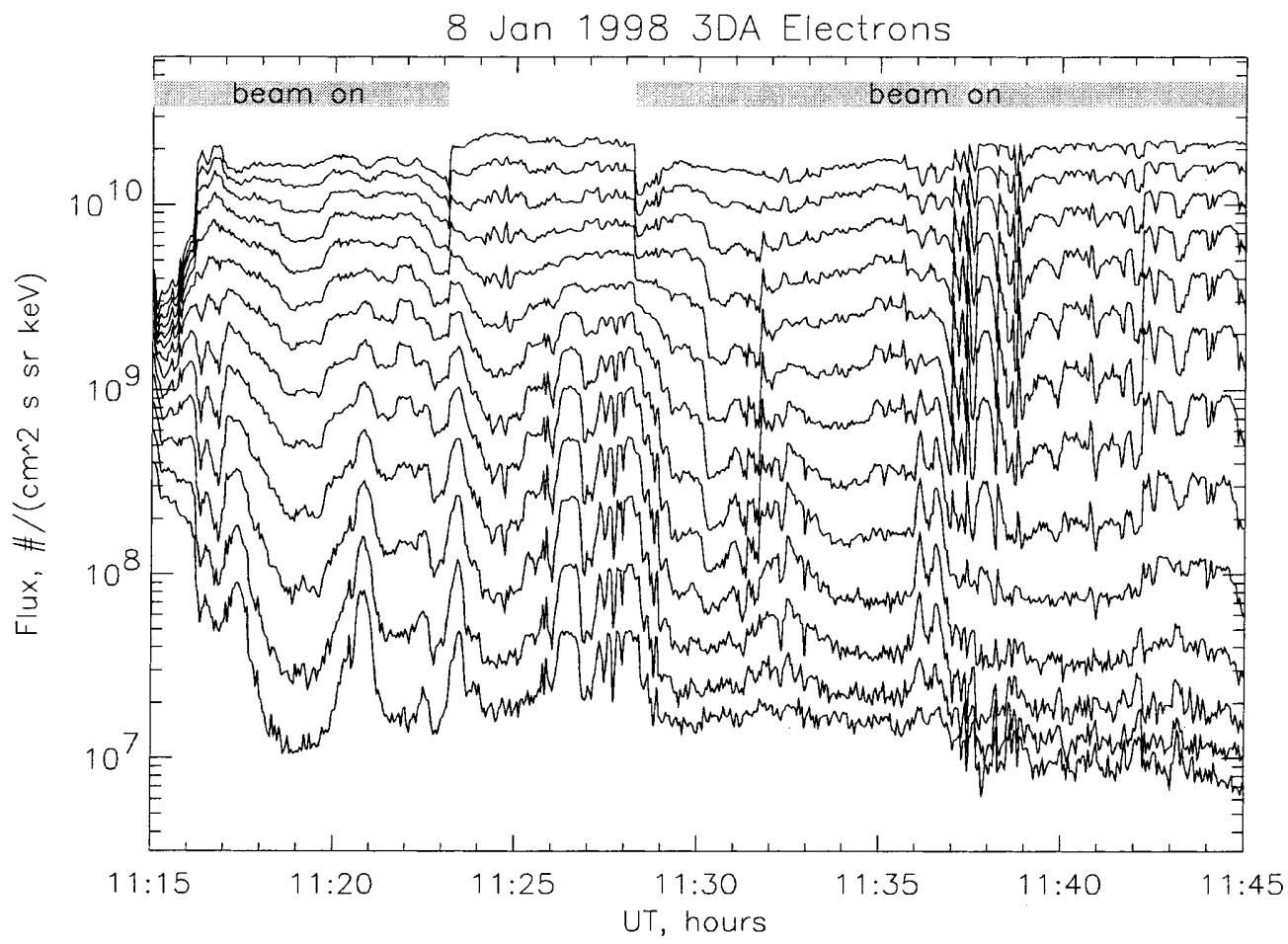

Fig. 6. Differential electron number fluxes measured by 3DA on January 8, 1998. The traces correspond to 13 logarithmically spaced energy channels between $7.5 \mathrm{eV}$ (top trace) and $98.8 \mathrm{eV}$ (lowest trace)

time interval is characterised by strong and highly variable electron fluxes, remnants of which appear as a flux drop-out after 11:15 UT. After 11:16 UT the spacecraft encounters a more uniform plasma environment. The fluxes shown are spin-averaged fluxes and are based on preliminary calibration information. The plasma density expected in this region ranges from $\approx 3$ to $\approx 10 \mathrm{~cm}^{-3}$ according to the statistical evaluation of ISEE-1 data by Escoubet et al. (1997).

The effect of turning off the ion beam between 11:23 and 11:28 UT is clearly visible in the upper traces of the plot for the lower energy channels. As expected, the flux is higher when the beam is off and the spacecraft potential is presumably more positive. Then more photo-electrons return to the spacecraft and the detector, and in addition the ambient electrons are accelerated in the potential well. The distribution functions immediately before and after beam turn-on and turn-off at 11:23 and 11:28 UT are plotted in Fig. 7. The distribution function measured at the lowest energy increases by about $60 \%$ when the ion beam is turned off, which gives an estimate of the error for density calculations based on data without potential control. All functions show deviations from a single Maxwellian distribution, which may indicate a superposition of photo-electrons to a Maxwellian population of plasma electrons. Above $\approx 20 \mathrm{eV}$ the spectra vary with time, whereas below this energy the values depend on the ion beam status only, which sets an upper energy limit at $\approx 20 \mathrm{eV}$ to the transition between photo and plasma electrons. Differences between the distribution functions with and without ion beam are, however, visible at all energies, as one would only expect for plasma electrons which are energised in the sheath around the spacecraft.
Distribution Functions at 11:23 and 11:28 UT

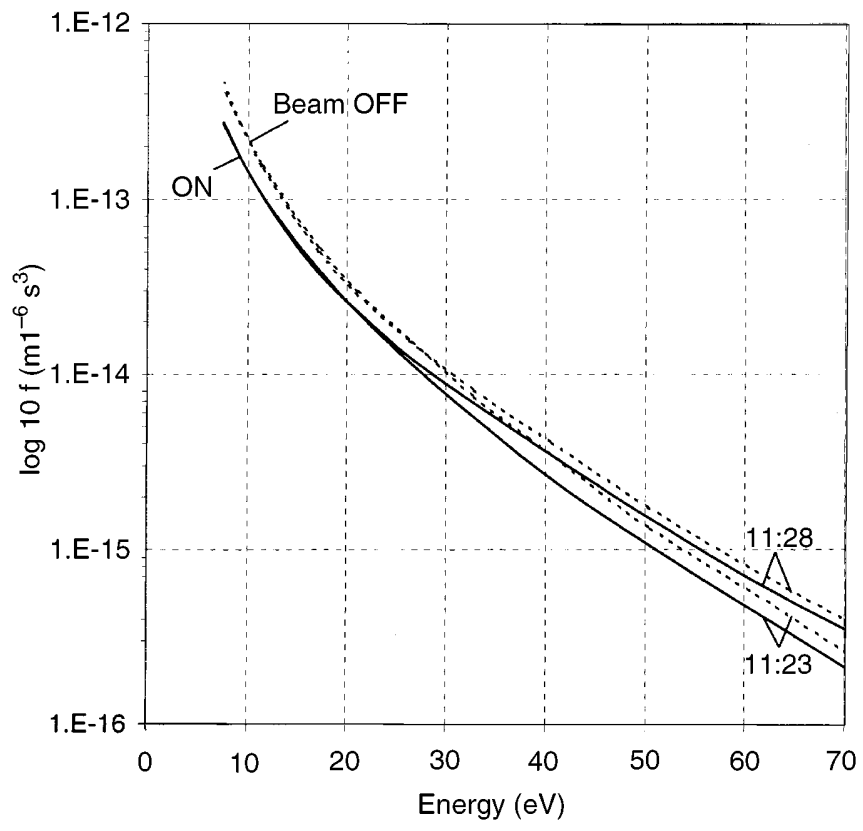

Fig. 7. Electron distribution functions measured by 3DA on January 8, 1998, at 11:23 and 11:28 UT with and without ion beam operation

Let us assume that photo-electrons do not contribute significantly to the counts, not even below $20 \mathrm{eV}$. In this case the common increase should be the result of the energisation of plasma electrons. In order to test this hypothesis the energy shift when the beam is turned on and off has been plotted versus distribution function in Fig. 8. One can see a nicely constant energy shift in the whole spectrum at 11:28 UT with an average of 


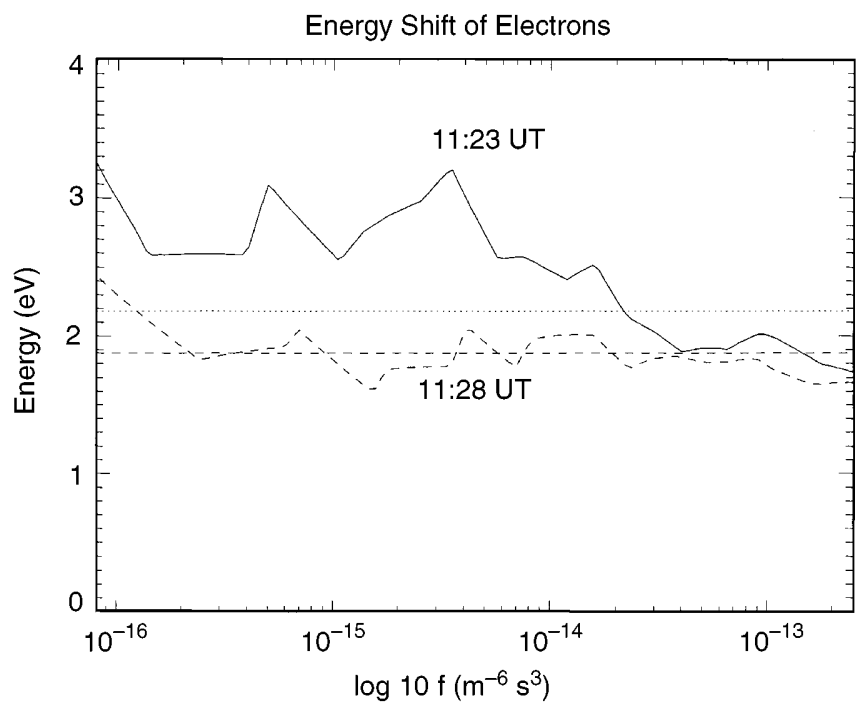

Fig. 8. Energy shift in electron distribution functions measured by 3DA on January 8, 1998, at ion beam turn-off (11:23) and turn-on (11:28 UT). The dashed and dotted lines represent the calculated mean values for 11:28 UT and for both times, respectively

$\approx 1.9 \mathrm{eV}$, suggesting that the spacecraft potential has changed by the same voltage when the ion beam was switched on. At 11:23 UT the energy shift for $f>4 \times 10^{-14} \mathrm{~m}^{-6} \mathrm{~s}^{3}$ (corresponding to energies below $\approx 20 \mathrm{eV}$ ) is similar, but increases slightly towards higher energies. Upon closer inspection of the fluxes around 11.23 UT one finds a time variation of the plasma electron flux which is superposed on the transition due to the ion beam and may be responsible for the apparent energy dependence. The energy shift averaged over both times is $\approx 2.2 \mathrm{eV}$.

Applying the usual approximations of distribution functions by Maxwellians and the coefficients for photoelectron current density as a function of the spacecraft potential given by Escoubet et al. (1997) one can calculate the effect of the ion beam emission for a given plasma density $n$. The following parameters of the Equator-S spacecraft were inserted: an almost cylindrical shape with $1.65 \mathrm{~m}$ diameter and a height of $1.16 \mathrm{~m}$, resulting in a surface area of $10.3 \mathrm{~m}^{2}$ and a projected sunlit area of $1.9 \mathrm{~m}^{2}$. Size and surface materials (Mylar and solar panels covered by indium-tin oxide) are similar to ISEE-1, for which the numerical results by Escoubet et al. (1997) are applicable. For a plasma electron thermal energy of $10 \mathrm{eV}$ (as measured by 3DA) a decrease of the spacecraft-plasma potential difference by 1.9 or $2.2 \mathrm{~V}$ corresponds to densities $n=7.5$ or $6.3 \mathrm{~cm}^{-3}$, respectively, which are typical values for this region. The ion beam reduces the spacecraft potential from 4.3 or $4.5 \mathrm{~V}$, respectively, to $2.3 \mathrm{~V}$. By applying another empirical relation between density and potential by Pedersen (1995) the absolute spacecraft potential remains the same, but the derived densities decrease by a factor of 1.2. The sensitivity of the potential shift $\Delta V$ on density is $\approx 4 \mathrm{~cm}^{-3} \mathrm{~V}^{-1}$ at this working point; other examples are $\left(\Delta V=5 \mathrm{~V}: n=1.6 \mathrm{~cm}^{-3}, \Delta V=1 \mathrm{~V}\right.$ : $\left.n=18 \mathrm{~cm}^{-3}\right)$.
As measurements by spacecraft in different regions often suggest strong photo-electron contamination up to several tens of eVs, one might argue that photoelectrons are responsible for the steep part of the spectra in Fig. 7, which would contradict the assumption made earlier. A look at Fig. 6, however, tells us that the flux at the lowest energy still varies by a factor of 2.5 during beam operation, when spacecraft potential control is in effect, whereas photo-electrons would produce constant count rates. By applying the model described already we find that a variation of density by a factor of 2.5 would limit the uncontrolled potential variations in this environment to $\approx 1.5 \mathrm{~V}$, and the residual variations with active control to $<0.5 \mathrm{~V}$. Moreover, the fluxes measured during the drop-out at 11:15 UT are lower than at 11:23 and 11:28 UT by a factor of 4 . All this suggests that the majority of electrons measured between 7.5 and $98.8 \mathrm{eV}$ and shown in Fig. 7 cannot be photo-electrons. We conclude that the observed variations of the electron distribution are consistent with the expected behaviour of the potential, and the ion beam for spacecraft potential control works as planned. The plasma density in this region was too high to show a dramatic decrease of the spacecraft potential due to the ion beam, but even under these conditions the electron data become significantly more trustworthy by a controlled spacecraft potential.

As size and surfaces of Equator-S are also similar to the Geotail spacecraft, the total flux of photo-electrons should be comparable and consequently, ion beams with the same current should have similar effects on Geotail and Equator-S. The consistency of the relevant parameters has been demonstrated at medium densities in the dayside equatorial magnetosphere. From these results it can be extrapolated that the ion beam would have been able to reduce potentials of several tens of volts in polar low density regions and in the lobes just as efficiently as the instrument aboard Geotail (Schmidt et al., 1995). A similar statement can be made for Cluster-II. The polar, $4 \times 19.6 \mathrm{R}_{\mathrm{E}}$ orbit of Cluster-II will also be even more suitable for potential control, particularly during the regular passes through the low density regions in the magnetospheric lobes. These spacecraft will have similar surface materials and the projected area to the Sun will be $\approx 2.9 \mathrm{~m}^{2}$, i.e. only by $53 \%$ higher than the area of Equator-S. Therefore ion beams with likewise higher currents will have the same effect. The instruments for Cluster-II have been designed for such currents.

\section{Conclusion}

The spacecraft potential control instrument PCD on Equator-S would have been very useful for the mission if the plasma instrument 3DA and the spacecraft had not failed early. After the seasonal rotation of the orbit towards the antisunward direction the spacecraft was expected to enter and leave the plasmasheet rather often due to the $23^{\circ}$ inclination to the ecliptic plane. This time interval, when spacecraft potential control would have 
been very useful to compensate for the effects of the varying plasma density, unfortunately comes too late. The technical experience from Equator-S is nevertheless also extremely important in order to secure the future application of spacecraft potential control aboard Cluster-II. The instrument PCD worked well, and ion emission was achieved with good stability throughout the whole routine operation phase. Overall, it was a technically successful test for the similar instrument on Cluster-II. Among the lessons learned for this mission is the observation that the operating voltage of the emitter should be monitored, and small deviations from the nominal value should be taken to trigger a preventive cleaning procedure. This procedure has already been implemented in the four similar instruments which are being built for Cluster-II.

Acknowledgements. The authors wish to thank all members of the instrument teams for their enthusiasm in building and operating the instruments. The authors are grateful for helpful comments by Dr. M. Friedrich.

The Editor-in-chief thanks E. Whipple and N. Dubouloz for their help in evaluating this paper.

\section{References}

Escoubet, C. P., A. Pedersen, and R. Schmidt, Density in the magnetosphere inferred from ISEE 1 spacecraft potential, J. Geophys. Res., 102(A8), 17 595-17 609, 1997.

Fehringer, M., F. Rüdenauer, and W. Steiger, Space-proven indium liquid metal ion emitters for ion microthruster applications, Proc. 33rd AIAA/ASME/SAE/ASEE Joint Propulsion Conference, AIAA-97-3057, 1-11, 1997.

Mahoney, J. F., A. Y. Yahiku, H. L. Daley, R. D. Moore, and J. Perel, Electrohydrodynamic ion source, J. Appl. Phys., 40, 5101-5106, 1969.

Moore, T. E., C. R. Chappell, M. O. Chandler, S. A. Fields, C. J. Pollock, D. L. Reasoner, D. T. Young, J. L. Burch, N. Eaker, J. H. Waite, Jr., D. J. McComas, J. E. Nordholdt, M. F. Thomsen, J. J. Berthelier, and R. Robson, The thermal ion dynamics experiment and plasma source instrument, Space Sci. Rev., 71, 409-458, 1995.

Olsen, R. C., The hidden ion population of the magnetosphere, $J$. Geophys. Res., 87(A5), 3481-3488, 1982.

Pedersen, A., Solar wind and magnetosphere plasma diagnostics by spacecraft electrostatic potential measurements, Ann. Geophysicae, 13(2), 118-129, 1995.

Pedersen, A., C. R. Chapell, K. Knott, and R. C. Olsen, Methods for keeping a conductive spacecraft near the plasma potential, in spacecraft plasma interactions and their influence on field and particle measurements, Proc. 17th ESLAB Symposium, ESA SP-198, 185-190, 1983.

Riedler, W., K. Torkar, F. Rüdenauer, M. Fehringer, A. Pedersen, R. Schmidt, R. J. L. Grard, H. Arends, B. T. Narheim, J. Trøim, R. Torbert, R. C. Olsen, E. Whipple, R. Goldstein, N. Valavanoglou, and Hua Zhao, Active spacecraft potential control, Space Science Rev., 79, 271-302, 1997.

Rüdenauer, F. G., W. Steiger, H. Studnicka, and P. Pollinger, Mass and energy spectra of a capillary - type In - liquid metal ion source, Int. J. Mass Spectrom. Ion Proc., 77, 63-74, 1987.

Schmidt, R., H. Arends, A. Pedersen, M. Fehringer, F. Rüdenauer, W. Steiger, B. T. Narheim, R. Svenes, K. Kvernsveen, K. Tsuruda, H. Hayakawa, M. Nakamura, W. Riedler, and K. Torkar, A novel medium-energy ion emitter for active spacecraft potential control, Rev. Sci. Inst., 64(8), 2293-2297, 1993.

Schmidt, R., H. Arends, A. Pedersen, F. Rüdenauer, M. Fehringer, B. T. Narheim, K. Svenes, K. Kvernsveen, K. Tsuruda, T. Mukai, H. Hayakawa, and M. Nakamura, Results from active spacecraft potential control on the Geotail spacecraft, J. Geophys. Res., 100(A9), 17 253-17 259, 1995.

Torkar, K., M. V. Veselov, V. V. Afonin, H. Arends, M. Fehringer, G. Fremuth, K. Fritzenwallner, Yc. I. Galperin, A. I. Kozlov, A. Pedersen, S. Perraut, W. Riedler, F. Rüdenauer, R. Schmidt, A. Smit, N. Valavanoglou, and L. V. Zinin, An experiment to study and control the langmuir sheath around INTERBALL, Ann. Geophysiae., 16, 1086-1096, 1998.

Whipple, E. C. Jr., Theory of the spherically symmetric photoelectron sheath: a thick sheath approximation and comparison with the ATS 6 observation of a potential barrier, J. Geophys. Res., 81(A4), 601-607, 1976.

Zhao, H, R. Schmidt, C. P. Escoubet, K. Torkar, and W. Riedler, Self-consistent determination of the electrostatic potential barrier due to the photoelectron sheath near a spacecraft, $J$. Geophys. Res., 101(A7), 15 653-15 659, 1996. 